\title{
Satisfying Convex Risk Limits by Trading
}

\author{
Kasper Larsen \\ Department of Accounting and Finance \\ Department of Mathematics and Computer Science \\ University of Southern Denmark \\ DK-5230 Odense M \\ kla@sam.sdu.dk \\ Traian Pirvu \\ Department of Mathematical Sciences \\ Carnegie Mellon University \\ Pittsburgh, PA \\ tpirvu+@andrew.cmu.edu \\ Steven E. Shreve ${ }^{\star}$ \\ Department of Mathematical Sciences \\ Carnegie Mellon University \\ Pittsburgh, PA \\ shreve@andrew.cmu.edu \\ Reha Tütüncü ${ }^{\star \star}$ \\ Department of Mathematical Sciences \\ Carnegie Mellon University \\ Pittsburgh, PA 15213 \\ reha+@andrew.cmu.edu
}

August 5, 2003

\begin{abstract}
A random variable, representing the final position of a trading strategy, is deemed acceptable if under each of a variety of probability measures its expectation dominates a floor associated with the measure. The set of random variables representing pre-final positions from which it is possible to trade to final acceptability is characterized. In particular, the set of initial capitals from which one can trade to final acceptability is shown to be a closed half-line $[\xi(0), \infty)$. Methods for computing $\xi(0)$ are provided, and the application of these ideas to derivative security pricing is developed.
\end{abstract}

JEL classification: G10

Mathematics Subject Classification (2000): 91B30, 60H30, 60G44

\footnotetext{
* Work supported by the National Science Foundation under grants DMS0103814 and DMS-0139911.

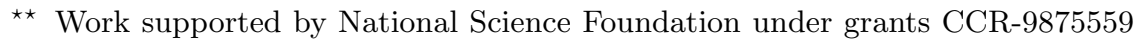
and DMS-0139911.
} 


\section{Introduction}

In their seminal paper on coherent risk measures, Artzner, Delbaen, Eber \& Heath [1] set forth axioms which should be satisfied by risk measures, and called risk measures satisfying these axioms coherent. They showed that in a finite probability space every coherent risk measure is characterized by a set of probability measures, the risk associated with a random loss being just its maximal expectation over the set of these measures. A position is acceptable in [1] if its risk measure is non-positive. Unfortunately, the most common risk measure, value-at-risk, does not satisfy the subadditivity axiom in [1] and hence is not coherent.

In a similar vein, Carr, Geman \& Madan [6] suggested that derivative security pricing should take into account a variety of measures, which they called valuation measures and stress measures. They defined acceptability of a random variable, representing potential gain from a derivative position, to mean that its expectation under each valuation measure is nonnegative and its expectation under each stress measure should be greater than or equal to a floor associated with that measure. When all the floors are zero, this notion of acceptability agrees with that of [1] (taking into account that one paper treats losses and the other treats gains). Carr et. al. [6] and Cerny \& Hodges [7] use these ideas to extend the concept of no-arbitrage pricing.

Föllmer \& Schied [15] consider convex risk measures, a class which includes the risk measures treated by both [1] and [6]. They show that these risk measures are like those of Carr, et. al. [6], except that their representation might require infinitely many measures and associated floors. Jaschke \& Küchler [17] further develop the properties of convex risk measures.

All these papers address a static situation: there is an initial date at which risk is measured and a final date at which random losses or gains are realized. The literature on dynamic risk measures is rather limited. In a series of papers [2], [3], [4], Artzner, Delbaen, Eber, Heath \& Ku build coherent risk measures on stochastic processes rather than on random variables. They obtain a representation result for such risk measures, discuss computational issues, and provide a multi-period extension of tail-value-at-risk. Basak \& Shapiro [5] consider a dynamic utility maximizer constrained to keep either value-at-risk or tail-value-at-risk below a threshold at all times. Wang [23] lists a set of axioms for dynamic risk measures and characterizes the class of measures satisfying his axioms. Cvitanić \& Karatzas [8] consider an agent trading in a market driven by Brownian motion who has insufficient initial capital to replicate a short position in a contingent claim he is forced to hold, and thus resorts to minimization of a coherent risk measure of hedge shortfall. Föllmer and Leukert [13] treat a similar situation, except asset prices are semimartingales, and the particular coherent risk measure to be minimized is the expected value of a convex function of hedge shortfall.

This paper considers a market with a semimartingale price process. We take as given a finite set of valuation and stress measures as in [6], which we call scenario measures. With each scenario measure there is an associated 
floor. These measures and floors determine if a random variable, representing the wealth of an agent at the final time, is acceptable. Section 2 describes the model, including the notion of scenario measures and floors.

Section 3 contains the central result of the paper, the characterization of random variables, representing the wealth of an agent at a time prior to the final time, from which the agent can trade to final acceptability. The representation is surprisingly simple and shows, in particular, that if one cannot form a martingale measure as a convex combination of scenario measures, then the final acceptability condition imposes no constraint on the initial wealth. Ruling out this case, we show in Section 4 that the set of initial positions from which final acceptability can be achieved is a closed half-line $[\xi(0), \infty)$. We provide primal and dual linear programs for computing $\xi(0)$.

In Section 5 we define buyer's and seller's prices for contingent claims. These are like utility indifference prices, except they are based on a concept of "risk-measure indifference." The buyer's and seller's prices thus obtained are within the interval of super-replication and sub-replication prices, and in the case of a complete market, coincide with the expected payoff under the martingale measure. Bounds for these prices are obtained in terms of the linear programs of Section 4.

Section 6 contains an auxiliary result needed to represent strategies which trade to final acceptability. In Section 7 we present a stochastic volatility model in which the scenario measures correspond to different levels to which the volatility reverts. In this example we provide a fairly explicit representation for strategies which trade to acceptability. Section 8 concludes the paper.

\section{The model}

\subsection{Asset price process and trading strategies}

We consider a market whose risk-free interest rate is equal to zero and which has one risky asset. The first assumption can be achieved without loss of generality by discounting asset prices. The inclusion of multiple risky assets in the model studied here requires little more than notational changes.

The price of our risky asset is a special semimartingale

$$
S_{t}=S_{0}+M_{t}+A_{t}, \quad 0 \leq t \leq T,
$$

defined on a probability space $(\Omega, \mathcal{F}, \mathbb{P})$. We denote by $\mathbb{E}$ the expectation operator under the "objective probability measure" $\mathbb{P}$. There is a filtration $\left\{\mathcal{F}_{t}\right\}_{0 \leq t \leq T}$ satisfying the usual conditions of right-continuity and augmentation by $\mathbb{P}$-null sets of $\mathcal{F}$. We assume $\mathcal{F}_{T}=\mathcal{F}$ and $\mathcal{F}_{0}$ contains only the $\mathbb{P}$-null subsets of $\mathcal{F}$ and their complements. Relative to this filtration, $M$ is a local martingale and $A$ is a predictable process of finite variation, both assumed to be càdlàg. The initial price $S_{0}>0$ is nonrandom, and $M_{0}=A_{0}=0$. We assume finiteness of the $\mathcal{H}^{2}$-norm of $S$, defined by

$$
\|S\|_{\mathcal{H}^{2}}^{2} \triangleq \mathbb{E}\left[[M]_{T}\right]+\mathbb{E}\left[\left(|A|_{T}\right)^{2}\right],
$$


where $|A|_{t}$ denotes the total variation of $A$ on $[0, t]$. This guarantees that $S$ is a square-integrable process and $M$ is a square-integrable martingale. The space of all special semimartingales with finite $\mathcal{H}^{2}$ norm is a Banach space (see [20] for these and following claims).

We define the linear space $\mathcal{H}^{2}(S)$ to be the set of all predictable processes $\pi$ satisfying

$$
\mathbb{E}\left[\int_{0}^{T} \pi_{u}^{2} d[M]_{u}\right]+\mathbb{E}\left[\left(\int_{0}^{T}\left|\pi_{u}\right| d|A|_{u}\right)^{2}\right]<\infty .
$$

Then the process

$$
(\pi \cdot S)_{t} \triangleq \int_{0}^{t} \pi_{u} d M_{u}+\int_{0}^{t} \pi_{u} d A_{u}
$$

is a special semimartingale, with local martingale part $(\pi \cdot M)_{t} \triangleq \int_{0}^{t} \pi_{u} d M_{u}$ and finite variation part $(\pi \cdot A)_{t} \triangleq \int_{0}^{t} \pi_{u} d A_{u}$. In fact, condition (2.2) implies $\pi \cdot S \in \mathcal{H}^{2}$ with $\|\pi \cdot S\|_{\mathcal{H}^{2}}^{2}$ equal to the quantity in (2.2). Furthermore,

$$
\mathbb{E}[\pi \cdot S]_{T}=\mathbb{E}\left[\int_{0}^{T} \pi_{u}^{2} d[M]_{u}\right]+\mathbb{E}\left[\sum_{0<u \leq t} \pi_{u}^{2}\left(\Delta A_{u}\right)^{2}\right] \leq\|\pi \cdot S\|_{\mathcal{H}^{2}}^{2}<\infty .
$$

See Dybvig \& Huang [10] for a discussion of the relationship between portfolio spaces of the form $\mathcal{H}^{2}(S)$ and those which are defined by the weaker condition (4.10) below in place of (2.2).

For any process $Z$, we define $\bar{Z}_{t} \triangleq \sup _{0 \leq u \leq t}\left|Z_{u}\right|$. The Davis-BurkholderGundy inequalities assert the existence of universal constants $K_{1}$ and $K_{2}$ such that every local martingale $Z$ satisfies

$$
\mathbb{E} \bar{Z}_{T} \leq K_{1} \mathbb{E}\left([Z]_{T}^{1 / 2}\right), \quad \mathbb{E} \bar{Z}_{T}^{2} \leq K_{2} \mathbb{E}[Z]_{T} .
$$

Therefore, for $\pi \in \mathcal{H}^{2}(S)$, we have

$$
\begin{aligned}
& \mathbb{E}\left[(\overline{\pi \cdot M})_{T}\right] \leq K_{1} \mathbb{E}\left[\left(\int_{0}^{T} \pi_{u}^{2} d[M]_{u}\right)^{1 / 2}\right]<\infty, \\
& \mathbb{E}\left[(\overline{\pi \cdot M})_{T}^{2}\right] \leq K_{2} \mathbb{E}\left[\int_{0}^{T} \pi_{u}^{2} d[M]_{u}\right]<\infty .
\end{aligned}
$$

Of course,

$$
\begin{aligned}
& \mathbb{E}\left[(\overline{\pi \cdot A})_{T}\right] \leq K_{1} \mathbb{E}\left[\left(\int_{0}^{T}\left|\pi_{u}\right| d|A|_{u}\right)\right]<\infty, \\
& \mathbb{E}\left[(\overline{\pi \cdot A})_{T}^{2}\right] \leq K_{2} \mathbb{E}\left[\left(\int_{0}^{T}\left|\pi_{u}\right| d|A|_{u}\right)^{2}\right]<\infty .
\end{aligned}
$$


It follows that for $\pi \in \mathcal{H}^{2}(S)$,

$$
\mathbb{E}\left[(\overline{\pi \cdot S})_{T}\right]<\infty, \quad \mathbb{E}\left[(\overline{\pi \cdot S})_{T}^{2}\right]<\infty .
$$

For $t \in[0, T]$, we may replace the initial time point 0 by $t$ in the above to obtain the analogous spaces $\mathcal{H}_{t}^{2}$ and $\mathcal{H}_{t}^{2}(S)$. Of course $\mathcal{H}_{0}^{2}=\mathcal{H}^{2}$.

Definition 1 For $t \in[0, T]$, a trading strategy on $[t, T]$ is a process $\pi$ in $\mathcal{H}_{t}^{2}(S)$. If $t=0$, we shall call $\pi$ simply a trading strategy. We denote by $L_{t}^{2}(\mathbb{P})$ the set of $\mathcal{F}_{t}$-measurable random variables with finite second moments under $\mathbb{P}$. If $\pi$ is a trading strategy on $[t, T]$ and $X \in L_{t}^{2}(\mathbb{P})$, we define the wealth process starting at $(t, X)$ generated by $\pi$ to be

$$
W_{u}^{t, X, \pi} \triangleq X+\int_{t}^{u} \pi_{v} d S_{v}, \quad t \leq u \leq T .
$$

Note that $W^{t, X, \pi}$ is in $\mathcal{H}_{t}^{2}$, and with $\left(\overline{W^{t, X, \pi}}\right)_{T} \triangleq \sup _{t \leq u \leq T}\left|W^{t, X, \pi}\right|_{u}$, we have

$$
\mathbb{E}\left[\left(\overline{W^{t, X, \pi}}\right)_{T}\right]<\infty, \quad \mathbb{E}\left[\left(\overline{W^{t, X, \pi}}\right)_{T}^{2}\right]<\infty .
$$

Definition 2 We denote by $\mathcal{M}^{a}$ (respectively, $\mathcal{M}_{t}^{a}$ ) the set of probability measures $\mathbb{Q}$ satisfying

(i) $\mathbb{Q} \ll \mathbb{P}$ and $\frac{d \mathbb{Q}}{d \mathbb{P}} \in L_{T}^{2}(\mathbb{P})$;

(ii) $S$ is a local martingale under $\mathbb{Q}$ on $[0, T]$ (respectively, on $[t, T]$ ).

We adopt the convention that $\mathcal{M}_{T}^{a}$ is the set of all probability measures $\mathbb{Q}$ satisfying (i). We denote by $\mathcal{M}^{e}$ (respectively, $\mathcal{M}_{t}^{e}$ ) the set of probability measures in $\mathcal{M}^{a}$ (respectively, $\mathcal{M}_{t}^{a}$ ) satisfying $\mathbb{P} \ll \mathbb{Q}$.

In order to rule out arbitrage, we make the following assumption.

Assumption $1 \mathcal{M}^{e} \neq \emptyset$.

Proposition 1 For any $t \in[0, T]$, any $\mathbb{Q} \in \mathcal{M}_{t}^{a}$, any $X \in L_{t}^{2}(\mathbb{P})$, and any trading strategy $\pi \in \mathcal{H}_{t}^{2}(S)$, the wealth process $W^{t, X, \pi}$ is a martingale on $[t, T]$ under $\mathbb{Q}$.

Proof: Because $S$ is a local martingale under every $\mathbb{Q} \in \mathcal{M}_{t}^{a}$ and $\pi \in$ $\mathcal{H}_{t}^{2}(S), W^{t, X, \pi}$ is a local martingale under every $\mathbb{Q} \in \mathcal{M}_{t}^{a}$ ([16], p. 45, (2.48)). To show that $W^{t, X, \pi}$ is a martingale under $\mathbb{Q} \in \mathcal{M}_{t}^{a}$, it suffices to show that $\mathbb{E}^{\mathbb{Q}}\left(\overline{W^{t, X, \pi}}\right)_{T}<\infty$. The Cauchy-Schwarz inequality implies

$$
\mathbb{E}^{\mathbb{Q}}\left(\overline{W^{t, X, \pi}}\right)_{T}=\mathbb{E}\left[\frac{d \mathbb{Q}}{d \mathbb{P}}\left(\overline{W^{t, X, \pi}}\right)_{T}\right] \leq\left\|\frac{d \mathbb{Q}}{d \mathbb{P}}\right\|_{L_{T}^{2}(\mathbb{P})}\left\|\left(\overline{W^{t, X, \pi}}\right)_{T}\right\|_{L_{T}^{2}(\mathbb{P})},
$$

which is finite by Definition 2(i) and the second part of (2.4).

Corollary 1 The prices process $S$ is a martingale under every $\mathbb{Q} \in \mathcal{M}^{a}$. 


\subsection{Scenario measures and floors}

We have a finite number of scenario probability measures $\mathbb{P}^{i}, i=1, \ldots, d$, each of which is absolutely continuous with respect to $\mathbb{P}$. Associated with each scenario measure $\mathbb{P}^{i}$ there is a floor $f^{i} \in \mathbb{R}$. For $i=1, \ldots, d$, we define

$$
Z_{T}^{i} \triangleq \frac{d \mathbb{P}^{i}}{d \mathbb{P}}, \quad Z_{t}^{i} \triangleq \mathbb{E}\left[Z_{T}^{i} \mid \mathcal{F}_{t}\right], 0 \leq t \leq T .
$$

Assumption $2 Z_{T}^{i} \in L_{T}^{2}(\mathbb{P}), i=1, \ldots, d$.

Under Assumption 2, the martingale $Z_{t}^{i}, 0 \leq t \leq T$, is square integrable under $\mathbb{P}$. In particular,

$$
\mathbb{E}\left[\left(\overline{Z^{i}}\right)_{T}^{2}\right] \leq K_{2} \mathbb{E}\left[Z^{i}\right]_{T}=K_{2} \mathbb{E}\left[\left(Z_{T}^{i}\right)^{2}\right]<\infty .
$$

Definition $3 A$ random variable $X \in L_{T}^{2}(\mathbb{P})$ is acceptable if $\mathbb{E}^{\mathbb{P}^{i}} X \geq f^{i}$ for $i=1, \ldots, d$. The set of all such random variables is denoted $\mathcal{A}_{T}$. For $t \in[0, T)$, a random variable $X \in L_{t}^{2}(\mathbb{P})$ is acceptable at time $t$ if there exists a trading strategy $\pi$ on $[t, T]$ satisfying $X+\int_{t}^{T} \pi_{u} d S_{u} \in \mathcal{A}_{T}$. The set of all time-t acceptable random variables is denoted $\mathcal{A}_{t}$.

Note that $X \in L_{T}^{2}(\mathbb{P})$ implies $\mathbb{E}^{\mathbb{P}^{i}} X$ is defined because of Assumption 2. Note also that for $0 \leq t \leq T, \mathcal{A}_{t}$ is a convex set, and $X \in \mathcal{A}_{t}$ and $Y \geq X$ almost surely under $\mathbb{P}$ imply that $Y \in \mathcal{A}_{t}$. Because one or more of the floors may be negative, the set $\mathcal{A}_{t}$ may fail to be a cone, i.e., $X \in \mathcal{A}_{t}$ and $\lambda \geq 0$ does not necessarily imply $\lambda X \in \mathcal{A}_{t}$. The set $\mathcal{A}_{0}$ is either $\mathbb{R}$, an open half-line extending to $\infty$, or a closed half-line extending to $\infty$. In the next section we rule out the second case and make an assumption which is necessary and sufficient to rule out the first case.

\section{Characterization of acceptable sets}

The convex hull $\operatorname{conv}\left\{\mathbb{P}^{i} ; i=1, \ldots, d\right\}$ is the set of probability measures of the form $\mathbb{Q}=\sum^{c} \lambda^{i} \mathbb{P}^{i}$, where the notation $\sum^{c}$ indicates convex combination, i.e., $\lambda_{i} \geq 0$ for every $i$ and $\sum_{i=1}^{d} \lambda_{i}=1$. If $X$ is acceptable and $\mathbb{Q} \in \operatorname{conv}\left\{\mathbb{P}^{i} ; i=1, \ldots, d\right\}$, then $\mathbb{E}^{\mathbb{Q}} X \geq f^{\mathbb{Q}}$, where

$$
f^{\mathbb{Q}} \triangleq \sup \left\{\sum^{c} \lambda^{i} f^{i} \mid \sum^{c} \lambda^{i} \mathbb{P}^{i}=\mathbb{Q}\right\} .
$$

For $t \in[0, T]$, we define

$$
\mathcal{C}_{t} \triangleq \operatorname{conv}\left\{\mathbb{P}^{i} \mid i=1, \ldots, d\right\} \cap \mathcal{M}_{t}^{a} .
$$

In particular, $\mathcal{C}_{T}=\operatorname{conv}\left\{\mathbb{P}^{i} \mid i=1, \ldots, d\right\}$. We further define

$$
\mathcal{X}_{t} \triangleq\left\{X \in L_{t}^{2}(\mathbb{P}) \mid \mathbb{E}^{\mathbb{Q}} X \geq f^{\mathbb{Q}} \forall \mathbb{Q} \in \mathcal{C}_{t}\right\} .
$$


Assumption 3 We assume that $\mathcal{C}_{0} \neq \emptyset$.

Because $\mathcal{M}_{t}^{a}$ is nondecreasing in $t$, so is $\mathcal{C}_{t}$. In particular, Assumption 3 guarantees that $\mathcal{C}_{t} \neq \emptyset$ for all $t \in[0, T]$.

We can now state the central result of this paper. The proof is given after two preparatory lemmas.

Theorem 1 For every $t \in[0, T]$, we have $\mathcal{X}_{t}=\mathcal{A}_{t}$.

For $t \in[0, T]$ and $i=1, \ldots, d$, we define a linear functional $F_{t}^{i}: \mathcal{H}_{t}^{2}(S) \rightarrow$ $\mathbb{R}$ by

$$
F_{t}^{i}(\pi) \triangleq \mathbb{E}^{\mathbb{P}^{i}} \int_{t}^{T} \pi_{u} d S_{u}=\mathbb{E}\left[Z_{T}^{i} \int_{t}^{T} \pi_{u} d S_{u}\right] \quad \forall \pi \in \mathcal{H}_{t}^{2}(S) .
$$

Hölder's inequality implies

$$
\left|F_{t}^{i}(\pi)\right| \leq\left\|Z_{T}^{i}\right\|_{L_{T}^{2}(\mathbb{P})} \cdot\|\pi\|_{\mathcal{H}_{t}^{2}(S)} \quad \forall \pi \in \mathcal{H}_{t}^{2}(S) .
$$

Lemma 1 (Farkas) Let $\left(a^{1}, \ldots, a^{d}\right) \in \mathbb{R}^{d}$ be given. For $t \in[0, T]$, the system of inequalities

$$
F_{t}^{i}(\pi) \geq a^{i}, \quad i=1, \ldots, d,
$$

has a solution $\pi \in \mathcal{H}_{t}^{2}(S)$ if and only if the following implication is true:

$$
\sum^{c} \lambda^{i} F_{t}^{i}=0 \Rightarrow \sum^{c} \lambda^{i} a^{i} \leq 0 .
$$

Proof: If (3.5) has a solution $\pi \in \mathcal{H}_{t}^{2}(S)$, and for some $\left(\lambda^{1}, \ldots, \lambda^{d}\right) \in \mathbb{R}_{+}^{d}$ satisfying $\sum_{i=1}^{d} \lambda^{i}=1$ we have $\sum^{c} \lambda^{i} F_{t}^{i}=0$, then $\sum^{c} \lambda^{i} a^{i} \leq \sum^{c} \lambda^{i} F_{t}^{i}(\pi)=$ 0 , so implication (3.6) holds.

For the reverse implication, we establish the contrapositive. Assume there is no solution to (3.5). We construct a vector $\left(\lambda^{1}, \ldots, \lambda^{d}\right) \in \mathbb{R}_{+}^{d}$ such that $\sum_{i=1}^{d} \lambda^{i}=1, \sum^{c} \lambda^{i} F_{t}^{i}=0$ and $\sum^{c} \lambda^{i} a^{i}>0$. To do this, we consider the range space of $\left(F_{t}^{1}, \ldots, F_{t}^{d}\right)$ in $\mathbb{R}^{d}$, i.e.,

$$
L \triangleq\left\{\left(F_{t}^{1}(\pi), \ldots, F_{t}^{d}(\pi)\right) \mid \pi \in \mathcal{H}_{t}^{2}(S)\right\} .
$$

The failure of (3.5) to have a solution means that $\left(a^{1}, \ldots, a^{d}\right) \notin L-\mathbb{R}_{+}^{d}$, which is closed (Corollary 19.3.2 of [21]). Hence there exists a strongly separating hyperplane, i.e., a vector $\left(\lambda^{1}, \ldots, \lambda^{d}\right) \in \mathbb{R}^{d}$ such that

$$
\sum_{i=1}^{d} \lambda^{i} a^{i}>\sum_{i=1}^{d} \lambda^{i}\left(F_{t}^{i}(\pi)-c^{i}\right) \quad \forall \pi \in \mathcal{H}_{t}^{2}(S), \quad \forall\left(c^{1}, \ldots, c^{d}\right) \in \mathbb{R}_{+}^{d} .
$$

Taking $\pi \equiv 0$ and $c^{i}$ large, we see that $\lambda^{i} \geq 0$ for every $i$. Taking $\pi \equiv 0$ and $c^{i}=0$ for every $i$, we see that $\sum_{i=1}^{d} \lambda^{i} a^{i}>0$. In particular, $\left(\lambda^{1}, \ldots, \lambda^{d}\right)$ is 
not the zero vector, and we can normalize it so $\sum_{i=1}^{d} \lambda^{i}=1$, as desired. Finally, taking $c^{i}=0$ for every $i$, we obtain

$$
\sum_{i=1}^{n} \lambda^{i} a^{i}>\sum_{i=1}^{d} \lambda^{i} F_{t}^{i}(\pi) \quad \forall \pi \in \mathcal{H}_{t}^{2}(S) .
$$

Since $F_{t}^{i}$ is linear, this implies $\sum_{i=1}^{d} \lambda^{i} F_{t}^{i}=0$.

Lemma 2 Let $t \in[0, T]$ be given. Then $\sum^{c} \lambda^{i} F_{t}^{i}=0$ if and only if the measure $\mathbb{Q} \triangleq \sum^{c} \lambda^{i} \mathbb{P}^{i}$ is in $\mathcal{M}_{t}^{a}$.

Proof: It is clear that $\mathbb{Q} \ll \mathbb{P}$ for any $\mathbb{Q}$ of the form $\mathbb{Q}=\sum^{c} \lambda^{i} \mathbb{P}^{i}$. Furthermore, because each $Z^{i} \in L^{2}(\mathbb{P}), d \mathbb{Q} / d \mathbb{P}$ also belongs to this set. Thus $\mathbb{Q} \triangleq \sum^{c} \lambda^{i} \mathbb{P}^{i} \in \mathcal{M}_{t}^{a}$ if and only if $S$ is a martingale on $[t, T]$ under $\mathbb{Q}$.

We first assume that $\sum^{c} \lambda^{i} F_{t}^{i}=0$ and prove the martingale property for $S$. Chose $t \leq u_{1} \leq u_{2} \leq T$ and $A \in \mathcal{F}_{u_{1}}$. Define $\pi_{v}=\mathbb{I}_{\left(u_{1}, u_{2}\right]}(v) \mathbb{I}_{A}$, which is predictable because it is left-continuous, and hence is in $\mathcal{H}_{t}^{2}(S)$. Then

$$
\begin{aligned}
\mathbb{E}^{\mathbb{Q}}\left[\mathbb{I}_{A} S_{u_{2}}\right]-\mathbb{E}^{\mathbb{Q}}\left[\mathbb{I}_{A} S_{u_{1}}\right] & =\sum^{c} \mathbb{E}\left[\lambda^{i} Z_{T}^{i}\left(S_{u_{2}}-S_{u_{1}}\right) \mathbb{I}_{A}\right] \\
& =\sum^{c} \lambda^{i} \mathbb{E}^{\mathbb{P}^{i}} \int_{t}^{T} \pi_{u} d S_{u} \\
& =\sum^{c} \lambda^{i} F_{t}(\pi)=0 .
\end{aligned}
$$

This shows that $\mathbb{E}^{\mathbb{Q}}\left[S_{u_{2}} \mid \mathcal{F}_{u_{1}}\right]=S_{u_{1}}$.

For the converse, suppose $S$ is a martingale under $\mathbb{Q}$. Then so is $\int_{t}^{u} \pi_{v} d S_{v}$, $t \leq u \leq T$, for every $\pi \in \mathcal{H}_{t}^{2}(S)$ (Proposition 1). It follows that

$$
\sum^{c} \lambda^{i} F_{t}^{i}(\pi)=\sum{ }^{c} \lambda^{i} \mathbb{E}^{\mathbb{P}^{i}} \int_{t}^{T} \pi_{v} d S_{v}=\mathbb{E}^{\mathbb{Q}} \int_{t}^{T} \pi_{v} d S_{v}=0 \forall \pi \in \mathcal{H}_{t}^{2}(S) .
$$

Proof of Theorem 1: The theorem requires a proof only for $t \in[0, T)$. For such a $t$, let $X \in \mathcal{A}_{t}$ be given. We can thus find $\pi \in \mathcal{H}_{t}^{2}(S)$ satisfying

$$
\mathbb{E}^{\mathbb{P}^{i}}\left[X+\int_{t}^{T} \pi_{v} d S_{v}\right] \geq f^{i}, \quad i=1, \ldots, d .
$$

Therefore, for any $\mathbb{Q} \in \mathcal{C}_{t}, \mathbb{E}^{\mathbb{Q}} X=\mathbb{E}^{\mathbb{Q}}\left[X+\int_{t}^{T} \pi_{v} d S_{v}\right] \geq f^{\mathbb{Q}}$, and hence $X \in \mathcal{X}_{t}$.

For the reverse containment, we begin with $X \in \mathcal{X}_{t}$. We must prove existence of $\pi \in \mathcal{H}_{t}^{2}(S)$ satisfying (3.8), i.e., satisfying $F_{t}^{i}(\pi) \geq a^{i}, i=$ $1, \ldots, d$, where $a^{i}=f^{i}-\mathbb{E}^{P^{i}} X$. According to Lemma 1 , it suffices to verify implication (3.6). Assume that $\left(\lambda^{1}, \cdots, \lambda^{d}\right)$ have been found such that $\sum^{c} \lambda^{i} F_{t}^{i}=0$. Then the probability measure $\mathbb{Q}$ defined in Lemma 2 is in $\mathcal{M}_{t}^{a}$. By virtue of its membership in $\mathcal{X}_{t}$, the random variable $X$ satisfies $\mathbb{E}^{\mathbb{Q}} X \geq f^{\mathbb{Q}}$. Therefore,

$$
\sum^{c} \lambda^{i} a^{i}=\sum^{c} \lambda^{i} f^{i}-\sum^{c} \lambda^{i} \mathbb{E}^{\mathbb{P}^{i}} X \leq f^{\mathbb{Q}}-\mathbb{E}^{\mathbb{Q}} X \leq 0 .
$$




\section{The acceptance set at time zero}

According to Theorem 1, the acceptance set at time zero is

$$
\begin{aligned}
\mathcal{A}_{0} & \triangleq\left\{x \in \mathbb{R} \mid \text { for some } \pi \in \mathcal{H}^{2}(S), F_{0}^{i}(\pi) \geq f^{i}-x, i=1, \ldots, d\right\} \\
& =\mathcal{X}_{0}=[\xi(0), \infty)
\end{aligned}
$$

where

$$
\xi(0) \triangleq \sup \left\{\sum^{c} \lambda^{i} f^{i} \mid \sum^{c} \lambda^{i} \mathbb{P}^{i} \in \mathcal{M}^{a}\right\}=\sup \left\{f^{\mathbb{Q}} \mid \mathbb{Q} \in \mathcal{C}_{0}\right\} .
$$

The supremum in (4.2) is not over the empty set because of Assumption 3.

We define

$$
\Lambda \triangleq\left\{\lambda=\left(\lambda^{1}, \ldots, \lambda^{d}\right) \in \mathbb{R}_{+}^{d} \mid \sum_{i=1}^{d} \lambda^{i}=1 \text { and } \sum_{i=1}^{d} \lambda^{i} \mathbb{P}^{i} \in \mathcal{M}^{a}\right\} .
$$

This is a closed set. To see this, let $\left\{\lambda_{n}\right\}=\left\{\left(\lambda_{n}^{1}, \ldots, \lambda_{n}^{d}\right)\right\}_{n=1}^{\infty}$ be a sequence in $\Lambda$ converging to a limit $\lambda=\left(\lambda^{1}, \ldots, \lambda^{n}\right)$. Let $\mathbb{Q}_{n} \triangleq \sum_{i=1}^{d} \lambda_{n}^{i} \mathbb{P}^{i}$. According to Corollary $1, S$ is a martingale under each $\mathbb{Q}_{n}$, i.e., for $0 \leq u \leq t \leq T$ and $A \in \mathcal{F}_{u}$,

$$
\sum_{i=1}^{d} \lambda_{n}^{i} \int_{A} S_{u} d \mathbb{P}^{i}=\int_{A} S_{u} d \mathbb{Q}_{n}=\int_{A} S_{t} d \mathbb{Q}_{n}=\sum_{i=1}^{d} \lambda_{n}^{i} \int_{A} S_{t} d \mathbb{P}^{i} .
$$

Letting $n \rightarrow \infty$, we see that $S$ has the martingale property under $\sum_{i=1}^{d} \lambda^{i} \mathbb{P}^{i}$, i.e., $\lambda \in \Lambda$.

Because $\Lambda$ is closed (and obviously bounded), the suprema in (4.2) are attained. In particular, $\xi(0)$ is finite, and the set $\Lambda(0)$ defined by (4.4) below is nonempty. We define

$$
\begin{aligned}
\Lambda(0) & \triangleq\left\{\lambda \in \Lambda \mid \sum^{c} \lambda^{i} f^{i}=\xi(0)\right\}, \\
I_{1} & \triangleq\left\{i \in\{1, \ldots, d\} \mid \exists \lambda \in \Lambda \text { such that } \lambda_{i}>0\right\} \\
I_{2} & \triangleq\left\{i \in\{1, \ldots, d\} \mid \exists \lambda \in \Lambda(0) \text { such that } \lambda_{i}>0\right\} .
\end{aligned}
$$

Like $\Lambda(0)$, the sets $I_{1}$ and $I_{2}$ are nonempty.

\subsection{Strongly relevant measures}

We call a scenario measure $\mathbb{P}^{i}$ for which $i \in I_{1}$ strongly relevant because it appears on the right-hand side of (4.2), even though it may not be used in a convex combination which attains the maximum in (4.2), i.e., even though it may not be in $I_{2}$. Assumption 3 is that there exists at least one strongly relevant measure. If $i \in I_{1}$ and the floor $f^{i}$ were replaced by a sufficiently large value, then $i$ would also be in $I_{2}$ and $\xi(0)$ would increase. (We will modify floors in the next section.) In contrast, for a scenario measure $\mathbb{P}^{i}$ for 
which $i \notin I_{1}$, no matter how large we make the associated floor $f^{i}$, the set $\mathcal{A}_{0}=[\xi(0), \infty)$ is unaffected. Starting from initial wealth $\xi(0)$, we can always find a trading strategy $\pi \in \mathcal{H}^{2}(S)$ such that $x+\int_{0}^{T} \pi_{u} d S_{u} \in \mathcal{A}_{T}$, regardless of the value $f^{i}$. However, the set of trading strategies which accomplish this depends the measures $\mathbb{P}^{i}$ and floors $f^{i}$ for $i \notin I_{1}$. Thus, although these are not strongly relevant, they are still relevant to the problem of acceptable trading.

Remark 1 It is perhaps surprising that a scenario measure which is not strongly relevant (at time zero) can become relevant at a later time. That is, there may be an index $i$ such that $\mathbb{P}^{i}$ never appears in a convex combination of scenario measures which is in $\mathcal{M}^{a}$, but $\mathbb{P}^{i}$ appears in a convex combination of scenario measures which is in $\mathcal{M}_{t}^{a}$ for some $t>0$. For example, suppose there are only two scenario measures. The first is $\mathbb{P}^{1} \in \mathcal{M}^{a}$. To construct the second, let $\mathbb{Q}$ be a probability measure equivalent to $\mathbb{P}^{1}$ such that $\mathbb{E}^{\mathbb{Q}} S_{\frac{T}{2}} \neq$ $S_{0}$. In particular, $S$ is not a $\mathbb{Q}$-martingale on $[0, T / 2]$. Define the second scenario measure $\mathbb{P}^{2}$ by

$$
\frac{d \mathbb{P}^{2}}{d \mathbb{P}^{1}} \triangleq \mathbb{E}^{\mathbb{P}^{1}}\left[\frac{d \mathbb{Q}}{d \mathbb{P}^{1}} \mid \mathcal{F}_{\frac{T}{2}}\right]
$$

Then

$$
\begin{aligned}
\mathbb{E}^{\sum{ }^{c} \lambda^{i} \mathbb{P}^{i}} S_{\frac{T}{2}} & =\sum^{c} \lambda^{i} \mathbb{E}^{\mathbb{P}^{i}} S_{\frac{T}{2}} \\
& =\lambda^{1} S_{0}+\lambda^{2} \mathbb{E}^{\mathbb{P}^{1}}\left[\frac{d \mathbb{P}^{2}}{d \mathbb{P}^{1}} S_{\frac{T}{2}}\right] \\
& =\lambda^{1} S_{0}+\lambda^{2} \mathbb{E}^{\mathbb{P}^{1}}\left[\frac{d \mathbb{Q}}{d \mathbb{P}^{1}} S_{\frac{T}{2}}\right] \\
& =\lambda^{1} S_{0}+\lambda^{2} \mathbb{E}^{\mathbb{Q}} S_{\frac{T}{2}},
\end{aligned}
$$

and this is different from $S_{0}$ unless $\lambda^{2}=0$. Therefore, $\sum^{c} \lambda^{i} \mathbb{P}^{i} \in \mathcal{M}^{a}$ implies $\lambda^{2}=0$, so $2 \notin I_{1}$. On the other hand, because $\frac{d \mathbb{P}^{2}}{d \mathbb{P}^{\mathbb{1}}}$ is $\mathcal{F}_{\frac{T}{2}}$-measurable, for $\frac{T}{2} \leq u \leq t \leq T$ and $A \in \mathcal{F}_{u}$, we have

$$
\int_{A} S_{u} d \mathbb{P}^{2}=\int_{A} S_{u} \frac{d \mathbb{P}^{2}}{d \mathbb{P}^{1}} d \mathbb{P}^{1}=\int_{A} S_{t} \frac{d \mathbb{P}^{2}}{d \mathbb{P}^{1}} d \mathbb{P}^{1}=\int_{A} S_{t} d \mathbb{P}^{2},
$$

so $\mathbb{P}^{2} \in \mathcal{M}_{\frac{T}{2}}^{a}$.

\subsection{Linear programs}

The number $\xi(0)$ is the value of the linear program

$$
\begin{array}{ll}
\operatorname{maximize} & \sum^{c} \lambda^{i} f^{i} \\
\text { subject to } & \sum^{c} \lambda^{i} \mathbb{P}^{i} \in \mathcal{M}^{a} .
\end{array}
$$


There is an associated dual program

(D)

$$
\begin{aligned}
\text { minimize } & y \\
\text { subject to } & \mathbb{E}^{\mathbb{P}^{i}} X \geq f^{i}, \quad i=1, \ldots, d, \\
& y-\mathbb{E}^{\mathbb{Q}} X \geq 0 \forall \mathbb{Q} \in \mathcal{M}^{a}, \\
& X \in L_{T}^{2}(\mathbb{P}) .
\end{aligned}
$$

To see that these programs stand in the weak duality relationship, let $\lambda=\left(\lambda^{1}, \ldots, \lambda^{d}\right)$ be a vector of nonnegative numbers summing to 1 so that $\sum^{c} \lambda^{i} \mathbb{P}^{i} \in \mathcal{M}^{a}$, i.e., $\lambda$ is a feasible solution for (P), and let $y \in \mathbb{R}$, $X \in L_{T}^{2}(\mathbb{P})$ be a feasible solution for (D). Then

$$
\sum^{c} \lambda^{i} f^{i} \leq \sum^{c} \lambda^{i} \mathbb{E}^{P^{i}} X=\mathbb{E}^{\sum{ }^{c} \lambda^{i} \mathbb{P}^{i}} X \leq y .
$$

The value in (D) may be written as $\inf _{X \in \mathcal{A}_{T}} \sup _{\mathbb{Q} \in \mathcal{M}^{a}} \mathbb{E}^{\mathbb{Q}} X$, and we have just seen that this quantity dominates $\xi(0)$, the value in $(\mathrm{P})$. In fact, these two quantities are equal (there is no duality gap), as the following theorem shows.

Theorem 2 Let $\lambda(0) \in \Lambda(0)$ be given, and define $\mathbb{Q}(0) \triangleq \sum^{c} \lambda^{i}(0) \mathbb{P}^{i}$. Let $\pi \in \mathcal{H}^{2}(S)$ be given so that $W^{0, \xi(0), \pi} \in \mathcal{A}_{T}$ (existence of $\pi$ is implied by (4.1)). For all $\mathbb{Q} \in \mathcal{M}^{a}$ and all $X \in \mathcal{A}_{T}$, we have

$$
\xi(0)=\mathbb{E}^{\mathbb{Q}} W_{T}^{0, \xi(0), \pi}=\mathbb{E}^{\mathbb{Q}(0)} W_{T}^{0, \xi(0), \pi} \leq \mathbb{E}^{\mathbb{Q}(0)} X .
$$

In particular,

$$
\xi(0)=\sup _{\mathbb{Q} \in \mathcal{M}^{a}} \inf _{X \in \mathcal{A}_{T}} \mathbb{E}^{\mathbb{Q}} X=\inf _{X \in \mathcal{A}_{T}} \sup _{\mathbb{Q} \in \mathcal{M}^{a}} \mathbb{E}^{\mathbb{Q}} X=\mathbb{E}^{\mathbb{Q}(0)} W_{T}^{0, \xi(0), \pi} .
$$

Proof: According to Proposition $1, W_{T}^{0, \xi(0), \pi}$ is a martingale for every $\mathbb{Q} \in \mathcal{M}^{a}$, which implies

$$
\xi(0)=\mathbb{E}^{\mathbb{Q}} W_{T}^{0, \xi(0), \pi}=\mathbb{E}^{\mathbb{Q}(0)} W_{T}^{0, \xi(0), \pi} .
$$

For $X \in \mathcal{A}_{T}$, we have

$$
\mathbb{E}^{\mathbb{Q}(0)} X=\sum^{c} \lambda^{i}(0) \mathbb{E}^{\mathbb{P}^{i}} X \geq \sum^{c} \lambda^{i}(0) f^{i}=\xi(0) .
$$

From (4.7) and (4.8) we obtain (4.5). Then (4.5) implies

$$
\begin{aligned}
\inf _{X \in \mathcal{A}_{T}} \sup _{\mathbb{Q} \in \mathcal{M}^{a}} \mathbb{E}^{\mathbb{Q}} X & \leq \sup _{\mathbb{Q} \in \mathcal{M}^{a}} \mathbb{E}^{\mathbb{Q}} W_{T}^{0, \xi(0), \pi} \\
& =\mathbb{E}^{\mathbb{Q}(0)} W_{T}^{0, \xi(0), \pi} \\
& =\inf _{X \in \mathcal{A}_{T}} \mathbb{E}^{\mathbb{Q}(0)} X \\
& \leq \sup _{\mathbb{Q} \in \mathcal{M}^{a}} \inf _{X \in \mathcal{A}_{T}} \mathbb{E}^{\mathbb{Q}} X .
\end{aligned}
$$

The reverse inequality $\inf _{X \in \mathcal{A}(T)} \sup _{Q \in \mathcal{M}^{a}} \mathbb{E}^{\mathbb{Q}} X \geq \sup _{Q \in \mathcal{M}^{a}} \inf _{X \in \mathcal{A}(T)} \mathbb{E}^{\mathbb{Q}} X$ is trivial. Therefore equality holds in (4.9), and combining this with (4.7), we obtain (4.6). 
Remark 2 The super-replicating price of a lower bounded random variable $X$ is the infimum of the set of $x$ such that there exists a trading strategy $\pi$ satisfying $W_{T}^{0, x, \pi} \geq X$ almost surely. Here $\pi$ is required to satisfy

$$
\int_{0}^{T} \pi_{u}^{2} d[M]_{u}<\infty, \quad \int_{0}^{T}\left|\pi_{u}\right| d|A|_{u}<\infty \quad \text { P-a. s. }
$$

rather than the stronger condition (2.2), and $\pi$ must also be such that $W_{t}^{0, x, \pi} \geq 0$ is lower bounded uniformly in $(t, \omega) \in[0, T] \times \Omega$. Kramkov [18] shows that the super-replicating price of $X$ is the supremum over all equivalent martingale measures $\mathbb{Q}$ of $\mathbb{E}^{\mathbb{Q}} X$. Kramkov's [18] set of equivalent martingale measures is larger than our $\mathcal{M}^{e}$ because [18] does not require the square-integrability of our Definition 2(i). However, Kramkov [19] shows that the square integrability restriction of Defintion $2(\mathrm{i})$ does not affect the supremum of $\mathbb{E}^{\mathbb{Q}} X$. Our set $\mathcal{M}^{a}$ may be larger than our set $\mathcal{M}^{e}$, but yields the same supremum because there exists a measure $\mathbb{Q}^{e} \in \mathcal{M}^{e}$ (Assumption 1 ) and for every $\mathbb{Q} \in \mathcal{M}^{a}$ and $\epsilon \in(0,1)$, the measure $\epsilon \mathbb{Q}^{e}+(1-\epsilon) \mathbb{Q}$ is in $\mathcal{M}^{e}$. Consequently, at least for lower bounded random variables $X \in \mathcal{A}_{T}$, the term $\sup _{\mathbb{Q} \in \mathcal{M}^{a}} \mathbb{E}^{\mathbb{Q}} X$ appearing in (4.6) is their super-replicating price.

Corollary 2 If $\pi \in \mathcal{H}^{2}(S)$ satisfies $W_{T}^{0, \xi(0), \pi} \in \mathcal{A}_{T}$, then

$$
\begin{aligned}
& \mathbb{E}^{\mathbb{P}^{i}} W_{T}^{0, \xi(0), \pi}=f^{i} \forall i \in I_{2}, \\
& \mathbb{E}^{\mathbb{Q}} W_{T}^{0, \xi(0), \pi}=f^{\mathbb{Q}} \forall \mathbb{Q} \in \mathcal{M}^{a} \cap \operatorname{conv}\left\{\mathbb{P}^{i} ; i \in I_{2}\right\} .
\end{aligned}
$$

Proof: For $i \in I_{2}$, there exists $\mathbb{Q}=\sum^{c} \lambda^{j} \mathbb{P}^{j} \in \mathcal{M}^{a}$ with $\sum_{j=1}^{d} \lambda^{j} f^{j}=\xi(0)$ and $\lambda^{i}>0$. We have

$$
\sum^{c} \lambda^{j} f^{j}=\xi(0)=\mathbb{E}^{\mathbb{Q}} W_{T}^{0, \xi(0), \pi}=\sum^{c} \lambda^{j} \mathbb{E}^{\mathbb{P}^{j}} W_{T}^{0, \xi(0), \pi} \geq \sum^{c} \lambda^{j} f^{j},
$$

and since $\lambda^{i}>0$, we must have $\mathbb{E}^{\mathbb{P}^{i}} W_{T}^{0, \xi(0), \pi}=f^{i}$.

Now suppose $\mathbb{Q}=\sum^{c} \lambda^{i} \mathbb{P}^{i} \in \mathcal{M}^{a}$, where $\lambda^{i} \in I_{2}$ for all $i$ appearing in this convex combination. We have just established $\mathbb{E}^{\mathbb{P}^{i}} W_{T}^{0, \xi(0), \pi}=f^{i}$ for all $i$ in this convex combination, so

$$
\xi(0)=\mathbb{E}^{\mathbb{Q}} W_{T}^{0, \xi(0), \pi}=\sum^{c} \lambda^{i} \mathbb{E}^{\mathbb{P}^{i}} W_{T}^{0, \xi(0), \pi}=\sum^{c} \lambda^{i} f^{i} \leq f^{\mathbb{Q}} .
$$

Recalling the definition $(3.2)$ of $\mathcal{X}_{0}=[\xi(0), \infty)$, we see that $\xi(0) \geq f^{\mathbb{Q}}$. Equation (4.12) follows.

Corollary 2 implies that if we start at $\xi(0)$, the boundary point of $\mathcal{A}_{0}=$ $[\xi(0), \infty)$, then any wealth random variable $W_{T}^{0, \xi(0), \pi} \in \mathcal{A}_{T}$ we can attain at the final time will be on the boundary of $\mathcal{A}_{T}$ in the sense that there will be a binding constraint of the form (4.11) (recall that $I_{2} \neq \emptyset$ ). Indeed, one can show that $W_{t}^{0, \xi(0), \pi}$ will be on the boundary of $\mathcal{A}_{t}$ for all $t \in[0, T]$ in the sense that

$$
\mathbb{E}^{\mathbb{Q}} W_{t}^{0, \xi(0), \pi}=f^{\mathbb{Q}} \quad \forall \mathbb{Q} \in \mathcal{M}_{t}^{a} \cap \operatorname{conv}\left\{\mathbb{P}^{i} \mid i \in I_{2}\right\} .
$$




\section{Pricing contingent claims}

In this section we consider a contingent claim $C \in L_{T}^{2}(\mathbb{P})$. An agent who is short $\alpha$ units of this claim must make payment $\alpha C$ at time $T$. If $0 \leq t \leq T$ and $X \in L_{t}^{2}(\mathbb{P})$ is the value of the agent's portfolio at time $t$, then the agent will seek a trading strategy $\pi \in \mathcal{H}_{t}^{2}(S)$ such that

$$
X+\int_{t}^{T} \pi_{u} d S_{u}-\alpha C \in \mathcal{A}_{T} .
$$

The set of random variables $X \in L_{t}^{2}(\mathbb{P})$ for which such a trading strategy exists will be denoted $\mathcal{A}_{t}(\alpha)$. In particular, $\mathcal{A}_{T}(\alpha)$ consists of those random variables $X \in L_{T}^{2}(\mathbb{P})$ satisfying

$$
\mathbb{E}^{\mathbb{P}^{i}} X \geq f^{i}+\alpha \mathbb{E}^{\mathbb{P}^{i}} C, i=1, \ldots, d .
$$

For $0 \leq t<T, \mathcal{A}_{t}(\alpha)$ is the set of random variables $X \in L_{t}^{2}(\mathbb{P})$ for which there exists a trading strategy $\pi \in \mathcal{H}_{t}^{2}(S)$ satisfying $X+\int_{t}^{T} \pi_{u} d S_{u} \in \mathcal{A}_{T}(\alpha)$. Thus the sets $\mathcal{A}_{t}(\alpha)$ are of the same form as the sets $\mathcal{A}_{t}$ of Definition 3 with the floors $f^{i}$ in that definition replaced by $f^{i}(\alpha) \triangleq f^{i}+\alpha \mathbb{E}^{\mathbb{P}^{i}} C$. Following (3.1), for $\mathbb{Q} \in \operatorname{conv}\left\{\mathbb{P}^{i} \mid i=1, \ldots, d\right\}$ we define

$$
f^{\mathbb{Q}}(\alpha)=\sup \left\{\sum^{c} \lambda^{i} f^{i}(\alpha) \mid \sum^{c} \lambda^{i} \mathbb{P}^{i}=\mathbb{Q}\right\}=f^{\mathbb{Q}}(0)+\alpha \mathbb{E}^{\mathbb{Q}} C .
$$

Note that $f^{\mathbb{Q}}(0)$ was earlier called simply $f^{\mathbb{Q}}$. Following $(3.2)$ we also define

$$
\mathcal{X}_{t}(\alpha) \triangleq\left\{X \in L_{t}^{2}(\mathbb{P}) \mid \mathbb{E}^{\mathbb{Q}} X \geq f^{\mathbb{Q}}(\alpha) \forall \mathbb{Q} \in \mathcal{C}_{t}\right\} .
$$

Theorem 1 has the following corollary.

Corollary 3 For every $t \in[0, T]$, we have $\mathcal{X}_{t}(\alpha)=\mathcal{A}_{t}(\alpha)$.

The results of Section 4 imply that

$$
\mathcal{A}_{0}(\alpha)=\mathcal{X}_{0}(\alpha)=[\xi(\alpha), \infty)
$$

where

$$
\xi(\alpha) \triangleq \sup \left\{\sum^{c} \lambda^{i} f^{i}(\alpha) \mid \sum^{c} \lambda^{i} \mathbb{P}^{i} \in \mathcal{M}^{a}\right\}=\sup \left\{f^{\mathbb{Q}}(\alpha) \mid \mathbb{Q} \in \mathcal{C}_{0}\right\} .
$$

With $\Lambda$ defined by (4.3), we set

$$
\Lambda(\alpha) \triangleq\left\{\lambda \in \Lambda \mid \sum^{c} \lambda^{i} f^{i}=\xi(\alpha)\right\}
$$

An agent who does not hold a position in the contingent claim $C$ needs initial capital $\xi(0)$ in order to trade to an acceptable position at time $T$. If the agent is short $C$, she will instead need initial capital $\xi(1)$. If she is long $C$, she will need initial capital $\xi(-1)$. This leads to the following definitions. 
Definition 4 The seller's price of the contingent claim $C$ is

$$
p(1) \triangleq \xi(1)-\xi(0),
$$

and the buyer's price is

$$
-p(-1) \triangleq \xi(0)-\xi(-1) .
$$

Remark 3 If there is only one equivalent martingale measure $\mathbb{Q} \in \mathcal{M}^{e}$, the buyer's and seller's price are both $\mathbb{E}^{\mathbb{Q}} C$. To see this, note first of all that $\mathcal{M}^{a}$ must be the singleton $\{\mathbb{Q}\}$. If there were a second measure $\mathbb{Q}^{\prime}$ in $\mathcal{M}^{a}$, then $\frac{1}{2}\left(\mathbb{Q}+\mathbb{Q}^{\prime}\right)$ would be a second equivalent martingale measure. According to Assumption $3, \mathcal{C}_{0} \neq \emptyset$, and hence $\mathcal{C}_{0}=\{\mathbb{Q}\}$. It follows that $\xi(\alpha)=f^{\mathbb{Q}}(\alpha)=$ $f^{\mathbb{Q}}(0)+\alpha \mathbb{E}^{\mathbb{Q}} C$, so $p(1)=-p(-1)=\mathbb{E}^{\mathbb{Q}} C$.

Remark 4 In the case of multiple equivalent martingale measures, the quantity $\sup _{\mathbb{Q} \in \mathcal{M}^{e}} \mathbb{E}^{\mathbb{Q}} C$ (see Remark 2) dominates $p(1)$. Indeed,

$$
\begin{aligned}
\xi(1) & =\sup _{\mathbb{Q} \in \mathcal{C}_{0}}\left(f^{\mathbb{Q}}(0)+\mathbb{E}^{\mathbb{Q}} C\right) \\
& \leq \sup _{\mathbb{Q} \in \mathcal{C}_{0}} f^{\mathbb{Q}}(0)+\sup _{\mathbb{Q} \in \mathcal{C}_{0}} \mathbb{E}^{Q} C \\
& =\xi(0)+\sup _{\mathbb{Q} \in \mathcal{C}_{0}} \mathbb{E}^{\mathbb{Q}} C \\
& \leq \xi(0)+\sup _{\mathbb{Q} \in \mathcal{M}^{a}} \mathbb{E}^{\mathbb{Q}} C .
\end{aligned}
$$

Typically $\mathcal{C}_{0}$ is a much smaller set than $\mathcal{M}^{a}$, so the last inequality is strict, i.e., $p(1)=\xi(1)-\xi(0)$ is strictly smaller than the super-replicating price. The next theorem provides tighter bounds on the buyer's and seller's prices.

Theorem 3 We have

$$
\begin{aligned}
& \max _{\lambda \in \Lambda(0)} \sum^{c} \lambda^{i} \mathbb{E}^{\mathbb{P}^{i}} C \leq p(1) \leq \min _{\lambda \in \Lambda(1)} \sum^{c} \lambda^{i} \mathbb{E}^{\mathbb{P}^{i}} C \\
& \max _{\lambda \in \Lambda(-1)} \sum^{c} \lambda^{i} \mathbb{E}^{\mathbb{P}^{i}} C \leq-p(-1) \leq \min _{\lambda \in \Lambda(0)} \sum^{c} \lambda^{i} \mathbb{E}^{\mathbb{P}^{i}} C .
\end{aligned}
$$

Proof: We prove (5.3); the proof of (5.4) is analogous. For $\alpha \in \mathbb{R}$ and $\lambda \in \Lambda$, define $g(\alpha ; \lambda) \triangleq \sum^{c} \lambda^{i} f^{i}(\alpha)$. For each $\lambda$, the mapping $\alpha \mapsto g(\alpha ; \lambda)$ is linear. Therefore, $\xi(\alpha)=\max _{\lambda \in \Lambda} g(\alpha ; \lambda)$ is convex in $\alpha$. The subdifferential $\partial \xi(\alpha)$ is the closed interval $\left[\xi^{\prime}(\alpha-), \xi^{\prime}(\alpha+)\right]$. According to [9], Theorem I.III,

$$
\begin{aligned}
& \xi^{\prime}(\alpha-)=\min _{\lambda \in \Lambda(\alpha)} \sum^{c} \lambda^{i} \frac{d}{d \alpha} f^{i}(\alpha)=\min _{\lambda \in \Lambda(\alpha)} \sum^{c} \lambda^{i} \mathbb{E}^{\mathbb{P}^{i}} C, \\
& \xi^{\prime}(\alpha+)=\max _{\lambda \in \Lambda(\alpha)} \sum^{c} \lambda^{i} \frac{d}{d \alpha} f^{i}(\alpha)=\max _{\lambda \in \Lambda(\alpha)} \sum^{c} \lambda^{i} \mathbb{E}^{\mathbb{P}^{i}} C .
\end{aligned}
$$

The inequalities in (5.3) now follow from the convex function property $\xi^{\prime}(0+) \leq \xi(1)-\xi(0) \leq \xi^{\prime}(1-)$. 


\section{Representation of $F_{t}^{i}$}

For the construction of trading strategies in the final section, we will need the following representation of the functionals $F_{t}^{i}$ defined by (3.3).

Proposition 2 For $i=1, \ldots, d$, define the finite-variation processes

$$
Y_{u}^{i} \triangleq\left\langle Z^{i}, S\right\rangle_{u}+\int_{0}^{u} Z_{v-}^{i} d A_{v}, \quad 0 \leq u \leq T .
$$

For $t \in[0, T]$ and $\pi \in \mathcal{H}_{t}^{2}(S)$, the process

$$
Z_{u}^{i} \int_{t}^{u} \pi_{v} d S_{v}-\int_{t}^{u} \pi_{v} d Y_{v}^{i}, \quad t \leq u \leq T,
$$

is a $\mathbb{P}$-martingale on $[t, T]$. In particular

$$
F_{t}^{i}(\pi)=\mathbb{E}\left[Z_{T}^{i} \int_{t}^{T} \pi_{v} d S_{v}\right]=\mathbb{E} \int_{t}^{T} \pi_{v} d Y_{v}^{i} .
$$

Proof: We may extend $\pi$ by defining it to be zero on $[0, t)$, so that the expression in (6.2) becomes

$$
Z_{u}^{i} \int_{0}^{u} \pi_{v} d S_{v}-\int_{0}^{u} \pi_{v} d Y_{v}^{i}, \quad t \leq u \leq T .
$$

Thus it suffices to prove that the expression in (6.4) is a $\mathbb{P}$-martingale on $[0, T]$ for every $\pi \in \mathcal{H}^{2}(S)$.

For $\pi \in \mathcal{H}^{2}(S)$, the product rule implies

$$
\begin{aligned}
Z_{u}^{i}(\pi \cdot S)_{u}= & \int_{0}^{u} Z_{v-}^{i} \pi_{v} d M_{v}+\int_{0}^{u} Z_{v-}^{i} \pi_{v} d A_{v} \\
& +\int_{0}^{u}(\pi \cdot S)_{v-} d Z_{v}^{i}+\int_{0}^{u} \pi_{v} d\left[Z^{i}, S\right]_{v} .
\end{aligned}
$$

The first and third terms on the right-hand side are martingales, which we establish by showing that their maxima have finite expectations. Because the integrators in these terms are martingales, the following bounds imply that the integrals are local martingales $([16]$, p. $45,(2.48))$. We can then apply the dominated convergence theorem to a localization of these processes to conclude they are martingales. For the first term we have the bound

$$
\begin{aligned}
\mathbb{E} \sup _{0 \leq u \leq T}\left|\int_{0}^{u} Z_{v-}^{i} \pi_{v} d M_{v}\right| & \leq K_{1} \mathbb{E}\left[\left(\int_{0}^{T}\left(Z_{v-}^{i} \pi_{v}\right)^{2} d[M]_{v}\right)^{1 / 2}\right] \\
& \leq K_{1} \mathbb{E}\left[\left(\overline{Z^{i}}\right)_{T}\left(\int_{0}^{t} \pi_{v}^{2} d[M]_{v}\right)^{1 / 2}\right] \\
& \leq K_{1}\left(\mathbb{E}\left[\left(\overline{Z^{i}}\right)_{T}^{2}\right]\right)^{1 / 2}\left(\mathbb{E} \int_{0}^{T} \pi_{v}^{2} d[M]_{v}\right)^{1 / 2}
\end{aligned}
$$


which is finite by (2.5) and (2.2). For the third term on the right-hand side of (6.5), we observe that

$$
\begin{aligned}
\mathbb{E} \sup _{0 \leq u \leq T}\left|\int_{0}^{u}(\pi \cdot S)_{v-} d Z_{v}^{i}\right| & \leq K_{1} \mathbb{E}\left[\left(\int_{0}^{T}(\pi \cdot S)_{v-}^{2} d\left[Z^{i}\right]_{v}\right)^{1 / 2}\right] \\
& \leq K_{1} \mathbb{E}\left[(\overline{\pi \cdot S})_{T}\left(\left[Z^{i}\right]_{T}\right)^{1 / 2}\right] \\
& \leq K_{1}\left(\mathbb{E}\left[(\overline{\pi \cdot S})_{T}^{2}\right]\right)^{1 / 2}\left(\mathbb{E}\left[Z^{i}\right]_{T}\right)^{1 / 2}
\end{aligned}
$$

which is finite by (2.3) and (2.5).

The Kunita-Watanabe inequality implies that because both $\left[Z^{i}\right]$ and $[\pi \cdot S]$ are integrable, the compensated process $\left[Z^{i}, \pi \cdot S\right]-\left\langle Z^{i}, \pi \cdot S\right\rangle$ is a $\mathbb{P}$-martingale, not just a local martingale. However, $\left[Z^{i}, \pi \cdot S\right]-\left\langle Z^{i}, \pi \cdot S\right\rangle=$ $\pi \cdot\left(\left[Z^{i}, S\right]-\left\langle Z^{i}, S\right\rangle\right)$. We may rewrite $(6.5)$ as

$$
\begin{aligned}
& Z_{u}^{i} \int_{0}^{u} \pi_{v} d S_{v}-\int_{0}^{u} \pi_{v} d Y_{v}^{i} \\
& =\int_{0}^{u} Z_{v-}^{i} \pi_{v} d M_{v}+\int_{0}^{u}(\pi \cdot S)_{v-} d Z_{v}^{i}+\int_{0}^{u} \pi_{v} d\left(\left[Z^{i}, S\right]_{v}-\left\langle Z^{i}, S\right\rangle_{v}\right),
\end{aligned}
$$

and we have shown that all three integrals on the right-hand side are $\mathbb{P}$ martingales.

\section{Example}

The primary source of risk in financial markets is market risk, and the next greatest concern is probably volatility risk. The Standard Portfolio Analysis of Risk (SPAN) system used by the Chicago Mercantile Exchange [22], for example, explicitly addresses these two types of risk.

In our setting, all the scenario measures are absolutely continuous with respect to the objective measure $\mathbb{P}$. As a practical matter, this means that every scenario which is considered by our risk control method must have positive probability under $\mathbb{P}$. Thus, in order to capture volatility risk, we must build a model in which volatility can change under $\mathbb{P}$. This section describes such a model, found in Fleming \& Hernández-Hernández [12] and Fouque \& Tullie [14], and discusses the application of the previous theory to it.

We specialize (2.1) by assuming that

$$
\frac{d S_{t}}{S_{t}}=\mu d t+\sigma\left(V_{t}\right) d B_{t},
$$

where the state process $V_{t}$ is given by

$$
d V_{t}=\rho\left(\bar{V}-V_{t}\right) d t+d W_{t} .
$$


Here $B$ and $W$ are independent Brownian motions under the objective measure $\mathbb{P}, \bar{V} \in \mathbb{R}$ is a level to which $V$ reverts, the coefficient $\rho>0$ controls the speed of the reversion to this level, $\mu \in \mathbb{R}$ is the mean rate of return of the risky asset, and the function $\sigma: \mathbb{R} \rightarrow[b, c]$ transforms the state process $V_{t}$ into asset volatility $\sigma\left(V_{t}\right)$. We assume that $0<b<c<\infty$ and that $v \mapsto \sigma(v)$ is nondecreasing. Under these conditions, the volatility $\sigma(V)$ inherits the reversion property of $V$, reverting to level $\sigma(\bar{V})$. Boundedness of $\sigma$ guarantees $S \in \mathcal{H}^{2}$.

\subsection{Scenario measures}

To model volatility risk, we consider different scenarios corresponding to different volatility reversion levels. Indeed, we can posit another mean rate of return $\mu^{i}(t)$ for the risky asset and another level of reversion $\bar{V}^{i}$ for the state process. Here $\mu^{i}(t)$ is a bounded nonrandom function of $t$. Because the process

$$
\eta_{t}^{i} \triangleq \frac{\mu^{i}(t)-\mu}{\sigma\left(V_{t}\right)}
$$

is bounded, the Doleans-Dade exponential

$$
\mathcal{E}_{t}^{B}(\eta) \triangleq \exp \left\{\int_{0}^{t} \eta_{v}^{i} d B_{v}-\frac{1}{2} \int_{0}^{t} \eta_{v}^{2} d v\right\}
$$

is a martingale. Similarly,

$$
\mathcal{E}_{t}^{W}\left(\rho\left(\bar{V}^{i}-\bar{V}\right)\right) \triangleq \exp \left\{\rho\left(\bar{V}^{i}-\bar{V}\right) W_{t}-\frac{1}{2} \rho^{2}\left(\bar{V}^{i}-\bar{V}\right)^{2} t\right\}
$$

is also a martingale. We define the scenario measure $\mathbb{P}^{i}$ by

$$
\frac{d \mathbb{P}^{i}}{d \mathbb{P}} \triangleq Z_{T}^{i} \triangleq \mathcal{E}_{T}^{B}\left(\eta^{i}\right) \mathcal{E}_{T}^{W}\left(\left(\rho\left(\bar{V}^{i}-\bar{V}\right)\right),\right.
$$

under which

$$
B_{t}^{i} \triangleq-\int_{0}^{t} \eta_{v}^{i} d v+B_{t}, \quad W_{t}^{i} \triangleq-\rho\left(\bar{V}^{i}-\bar{V}\right) t+W_{t}
$$

are independent Brownian motions. We may rewrite (7.1), (7.2) so that under $\mathbb{P}^{i}$ we have the alternate model for asset and state processes

$$
\begin{aligned}
\frac{d S_{t}}{S_{t}} & =\mu^{i}(t) d t+\sigma\left(V_{t}\right) d B_{t}^{i}, \\
d V_{t} & =\rho\left(\bar{V}^{i}-V_{t}\right) d t+d W_{t}^{i} .
\end{aligned}
$$

We also have

$$
Z_{t}^{i} \triangleq \mathbb{E}\left[Z_{T}^{i} \mid \mathcal{F}_{t}\right]=\mathcal{E}_{t}^{B}\left(\eta^{i}\right) \mathcal{E}_{t}^{W}\left(\left(\rho\left(\bar{V}^{i}-\bar{V}\right)\right)\right.
$$


We consider this model with at least two scenario measures $\mathbb{P}^{1}$ and $\mathbb{P}^{2}$ corresponding to two levels of reversion $\bar{V}^{1}$ and $\bar{V}^{2}$ and to two nonrandom, bounded mean-return functions $\mu^{1}(t)$ and $\mu^{2}(t)$ related by

$$
\mu^{2}(t)=-\frac{\alpha \mu^{1}(t) \exp \left(\int_{t}^{T} \mu^{1}(v) d v\right)}{1-\alpha \exp \left(\int_{t}^{T} \mu^{1}(v) d v\right)}, 0 \leq t \leq T,
$$

where $\alpha \in(0,1)$ is a constant.

\subsection{Verification of assumptions}

We must verify Assumptions 1-3. Because $\eta^{i}$ is bounded and $\rho\left(\bar{V}^{i}-\bar{V}\right)$ is constant, all moments under $\mathbb{P}$ of $\mathcal{E}_{T}^{B}\left(\eta^{i}\right)$ and $\mathcal{E}_{T}^{W}\left(\rho\left(\bar{V}^{i}-\bar{V}\right)\right)$ are finite. Using Hölder's inequality, we obtain Assumption 2: $Z_{T}^{i} \in L_{T}^{2}(\mathbb{P}), i=1,2$.

Assumptions 1 and 3 follow from the next proposition.

Proposition 3 The probability measure $\alpha \mathbb{P}^{1}+(1-\alpha) \mathbb{P}^{2}$ is in $\mathcal{M}^{e}$ and hence in $\mathcal{M}^{a}$ and $\mathcal{C}_{0}$.

Proof: From (7.4) with $i=1$, we see that $\exp \left(-\int_{0}^{t} \mu^{1}(v) d v\right) S_{t}$ is a martingale under $\mathbb{P}^{1}$. Therefore,

$$
\mathbb{E}^{\mathbb{P}^{1}}\left[S_{T} \mid \mathcal{F}_{t}\right]=S_{t} \exp \left(\int_{t}^{T} \mu^{1}(v) d v\right) .
$$

Let us define

$$
Z_{t} \triangleq \frac{Z_{t}^{2}}{Z_{t}^{1}}=\mathcal{E}_{t}^{B^{1}}\left(\frac{\mu^{2}-\mu^{1}}{\sigma(V)}\right) \mathcal{E}_{t}^{W^{1}}\left(\rho\left(\bar{V}^{2}-\bar{V}^{1}\right)\right)=\mathbb{E}^{\mathbb{P}^{1}}\left[\frac{d \mathbb{P}^{2}}{d \mathbb{P}^{1}} \mid \mathcal{F}_{t}\right] .
$$

We set

and use (7.6) to compute

$$
M_{t}=Z_{t} S_{t}\left[1-\alpha \exp \left(\int_{t}^{T} \mu^{1}(v) d v\right)\right]
$$

$$
d M_{t}=M_{t}\left(\frac{\mu^{2}(t)-\mu^{1}(t)}{\sigma\left(V_{t}\right)}+\sigma\left(V_{t}\right)\right) d B_{t}^{1}+M_{t} \rho\left(\bar{V}^{2}-\bar{V}^{1}\right) d W_{t}^{1},
$$

which is a $\mathbb{P}^{1}$-martingale. Therefore,

$$
\begin{aligned}
\mathbb{E}^{\alpha \mathbb{P}^{1}+(1-\alpha) \mathbb{P}^{2}}\left[S_{T} \mid \mathcal{F}_{t}\right] & =\alpha \mathbb{E}^{\mathbb{P}^{1}}\left[S_{T} \mid \mathcal{F}_{t}\right]+(1-\alpha) \mathbb{E}^{\mathbb{P}^{2}}\left[S_{T} \mid \mathcal{F}_{t}\right] \\
& =\alpha S_{t} \exp \left(\int_{t}^{T} \mu^{1}(v) d v\right)+\frac{1}{Z_{t}} \mathbb{E}^{\mathbb{P}^{1}}\left[(1-\alpha) Z_{T} S_{T} \mid \mathcal{F}_{t}\right] \\
& =\alpha S_{t} \exp \left(\int_{t}^{T} \mu^{1}(v) d v\right)+\frac{1}{Z_{t}} \mathbb{E}^{\mathbb{P}^{1}}\left[M_{T} \mid \mathcal{F}_{t}\right] \\
& =S_{t},
\end{aligned}
$$

where have obtained the second equality from (7.7) and the last from the $\mathbb{P}^{1}$-martingale property of $M$. 


\subsection{Portfolio representation}

We assume now that there are $d$ scenario measures of the type described in Subsection 7.1. Associated with each scenario measure $\mathbb{P}^{i}$ we have a floor $f^{i}$. This determines a set of random variables $\mathcal{A}_{T}$ which are acceptable at the final time (Definition 3). Now let $t \in[0, T)$ be fixed, and let $X \in \mathcal{A}_{t}$ be given. By the definition of $\mathcal{A}_{t}$, there is a trading strategy $\bar{\pi}$ on $[t, T]$ satisfying

$$
W_{T}^{t, X, \bar{\pi}} \triangleq X+\int_{t}^{T} \bar{\pi}_{v} d S_{v} \in \mathcal{A}_{T} .
$$

In this example, we provide a representation for such a trading strategy in terms of the processes $Y^{i}$ of (6.1). In the case at hand,

$$
Y_{u}^{i}=\int_{0}^{u} Z_{\theta}^{i} S_{\theta}\left(\mu+\eta_{\theta}^{i} \sigma\left(V_{\theta}\right)\right) d \theta .
$$

Recall that $\pi \in \mathcal{H}_{t}^{2}(S)$ if and only if $\pi$ is measurable with respect to the predictable $\sigma$-algebra on $[t, T] \times \Omega$ and

$$
\mathbb{E} \int_{t}^{T} \pi_{u}^{2} \sigma^{2}\left(V_{u}\right) S_{u}^{2} d u+\mathbb{E}\left[\left(\int_{t}^{T}\left|\mu \pi_{u}\right| S_{u} d u\right)^{2}\right]<\infty .
$$

Because $\sigma^{2}$ is bounded away from zero, the finiteness of $\mathbb{E} \int_{t}^{T} \pi_{u}^{2} \sigma^{2}\left(V_{u}\right) S_{u}^{2} d u$ implies the finiteness of $\mathbb{E}\left[\left(\int_{t}^{T}\left|\mu \pi_{u}\right| S_{u} d u\right)^{2}\right]$. We can thus regard $\mathcal{H}_{t}^{2}(S)$ as the Hilbert space of functions on $[t, T] \times \Omega$, measurable with respect to the predictable $\sigma$-algebra, and square-integrable with respect to the measure $d[S] \times d \mathbb{P}$. The inner product on this space is

$$
\left\langle\Xi^{i}, \Xi^{j}\right\rangle \triangleq \mathbb{E} \int_{t}^{T} \Xi_{u}^{i} \Xi_{u}^{j} d[S]_{u}=\mathbb{E} \int_{t}^{T} \Xi_{u}^{i} \Xi_{u}^{j} \sigma\left(V_{u}\right) S_{u}^{2} d u .
$$

For $i=1, \ldots, d$, we define the predictable process

$$
X_{u}^{i} \triangleq \frac{d Y_{u}^{i}}{d[S]_{u}}=\frac{Z_{u}^{i}\left(\mu+\eta_{u}^{i} \sigma\left(V_{u}\right)\right)}{\sigma^{2}\left(V_{u}\right) S_{u}}, t \leq u \leq T .
$$

Recall from (6.3) that

$$
F_{t}^{i}(\pi)=\mathbb{E} \int_{t}^{T} \pi_{u} d Y_{u}^{i}=\left\langle\pi, X^{i}\right\rangle \quad \forall \pi \in \mathcal{H}_{t}^{2}(S),
$$

and from (3.4) that

$$
\left|F_{t}^{i}(\pi)\right| \leq\left\|Z_{T}^{i}\right\|_{L_{T}^{2}(\mathbb{P})} \cdot\|\pi\|_{H_{t}^{2}(S)} \quad \forall \pi \in \mathcal{H}_{t}^{2}(S), i=1, \ldots, d .
$$

By the converse of Hölder's inequality ([11], (6.14), p. 181), the process $X^{i}$ must also be in $\mathcal{H}_{t}^{2}(S), i=1, \ldots, d$. It follows that any linear combination 
of these processes is in $\mathcal{H}_{t}^{2}(S)$. We show that such a linear combination can serve as a portfolio $\pi \in \mathcal{H}_{t}^{2}(S)$ satisfying $W_{T}^{t, X, \pi} \in \mathcal{A}_{T}$.

Note that $W_{T}^{t, X, \pi} \in \mathcal{A}_{T}$ is equivalent to

$$
\mathbb{E} \int_{t}^{T} \pi_{u} d Y_{u}^{i}=F_{t}^{i}(\pi) \geq a^{i}, i=1, \ldots, d,
$$

where $a^{i} \triangleq f^{i}-\mathbb{E}^{\mathbb{P}^{i}} X$. We define $a$ to be the $d$-dimensional column vector $a=\left(a^{1}, \ldots, a^{d}\right)^{t r}$. We further define the $d \times d$-matrix $A=\left(A^{i, j}\right)$ by

$$
A^{i, j} \triangleq\left\langle X^{i}, X^{j}\right\rangle, \quad \forall i, j=1, \ldots, d .
$$

Proposition 4 Let $t \in[0, T]$ be fixed and let $X \in \mathcal{A}_{t}$ be given. The vector inequality

$$
A x \geq a
$$

has a solution $x=\left(x^{1}, \ldots, x^{d}\right)^{t r}$. For any such solution $x$, the process

$$
\pi_{u} \triangleq \sum_{i=1}^{d} x^{i} X_{u}^{i}, t \leq u \leq T
$$

is in $\mathcal{H}_{t}^{2}(S)$ and satisfies $F_{t}^{i}(\pi) \geq a^{i}, i=1, \ldots, d$, or equivalently, $W_{T}^{t, X, \pi} \in$ $\mathcal{A}_{T}$.

Proof: The standard Farkas Lemma says that (7.12) has a solution if and only if the implication

$$
x \geq 0, x^{t r} A=0 \Rightarrow x^{t r} a \leq 0
$$

holds. We verify this implication. Suppose $x \geq 0$ and $x^{t r} A=0$. Then

$$
\mathbb{E} \int_{t}^{T}\left(\sum_{i=1}^{d} x^{i} X^{i}\right)^{2} d[S]_{u} d u=x^{\operatorname{tr}} A x=0 .
$$

In other words, $\sum_{i=1}^{d} x^{i} X_{u}^{i}=0$ for $d[S]$-almost every $u \in[t, T]$, almost surely. It follows from (7.8), (7.10) that

$\sum_{i=1}^{d} x^{i} a^{i} \leq \sum_{i=1}^{d} x^{i} F_{t}^{i}(\bar{\pi})=\mathbb{E} \sum_{i=1}^{d} x^{i} \int_{t}^{T} \bar{\pi}_{u} d Y_{u}^{i} \leq \mathbb{E} \int_{t}^{T} \bar{\pi}_{u}\left(\sum_{i=1}^{d} x^{i} X_{u}^{i}\right) d[S]_{u}=0$, and the implication (7.14) is established.

Let $x=\left(x^{1}, \ldots, x^{d}\right)^{\operatorname{tr}}$ be a solution to (7.12) and let $\pi$ be defined by (7.13). We compute for $i=1, \ldots, d$ :

$$
F_{t}^{i}(\pi)=\mathbb{E} \int_{t}^{T} \pi_{u} d Y_{u}^{i}=\mathbb{E} \int_{t}^{T}\left(\sum_{j=1}^{d} x^{j} X_{u}^{j}\right) X_{u}^{i} d[S]_{u}=\sum_{j=1}^{d} A^{i, j} x^{j} \geq a^{i}
$$

by (7.12). 


\section{Conclusion}

We have posited a finite set of scenario measures and associated floors, and have defined a random variable to be acceptable if its expectation under each scenario measure equals or exceeds the corresponding floor. We have examined the problem of beginning with a certain capital at time zero, or with a random capital at a later, pre-final time, and trading to a final acceptable random variable. We have characterized the set of pre-final random variables which permit such trading. When the pre-final time is time zero, the set of initial capitals which permit trading to an acceptable final random variable is a closed half-line. We have characterized the end-point of this half-line via primal and dual linear programs. We have also introduced the seller's (respectively, buyer's) price for a contingent claim at the initial time as the change in the initial capital needed in order for an agent to trade to an acceptable final random variable when the contingent claim is a liability (respectively, asset) in the agent's portfolio. We have provided bounds on these prices. Finally, in a stochastic volatility example we have derived a representation for trading strategies which achieve acceptability at the final time.

\section{References}

1. Artzner, P., Delbaen, F., Eber, J.M. \& Heath, D. (1999) Coherent measures of risk, Math. Finance 9, 203-228.

2. Artzner, P, Delbaen, F., Eber, J.-M., Heath, D. \& Ku, H. (2002) Coherent multiperiod risk measurement, working paper, Department of Mathematics, ETH-Zürich.

3. Artzner, P, Delbaen, F., Eber, J.-M., Heath, D. \& Ku, H. (2003) Coherent multiperiod risk adjusted values and Bellman's principle, working paper, Department of Mathematics, ETH-Zürich.

4. Artzner, P, Delbaen, F., Eber, J.-M., Heath, D. \& Ku, H. (2003) Multiperiod risk and coherent multiperiod risk measurement, working paper, Department of Mathematics, ETH-Zürich.

5. BAsak, S. \& Shapiro, A. (2001) Value-at-risk-based risk management: optimal policies and asset prices, Rev. Financial Studies 14, 371-405.

6. Carr, P., Geman, H. \& Madan, D. (2001) Pricing and hedging in incomplete markets, J. Financial Economics 62, 131-167.

7. Cerny, A. \& Hodges, S. (1998) The theory of no-good-deal pricing in financial markets, Financial Options Research Center, Warwick University, working paper.

8. Cvitanić, J. \& Karatzas, I. (1999) On dynamic measures of risk, Finance Stochastics 3, 451-482.

9. Danskin, J. M., The Theory of Max-Min and its Application to Weapon Allocation Problems, Springer, New York, 1967.

10. Dybvig, P. \& Huang, C. (1988) Nonnegative wealth, absence of arbitrage, and feasible consumption plans, Rev. Financial Studies 1, 377-401.

11. Folland, G, Real Analysis, Wiley, New York, 1984. 
12. Fleming, W. \& Hernández-Hernández, D. (2003) An optimal consumption model with stochastic volatility, Finance Stochastics 7, 245-262.

13. Föllmer, H. \& Leukert, P. (2000) Efficient hedging: cost versus shortfall risk, Finance Stochastics 4, 117-146.

14. Fouque, J.-P. \& Tullie, T. (2002) Variance reduction for Monte Carlo simulation in a stochastic volatility environment, Quantitative Finance 2, 2430.

15. Föllmer, H. \& Schied, A. Stochastic Finance, de Gruyter, Berlin, 2002.

16. JACOD, J. Calcul Stochastique et Problèmes de Martingales, Lecture Notes in Mathematics 714, Springer, Berlin, 1979.

17. Jaschke, S. \& Küchler, U. (2001) Coherent risk measures and good-deal bounds, Finance Stochastics 5, 181-200.

18. KRAmkov, D. (1996) Optional decomposition of supermartingales and hedging contingent claims in incomplete security markets, Probab. Theory Relat. Fields 105, 459-479.

19. KRAmkov, D. (1996) On closure of a family of martingale measures and an optional decomposition of supermartingales (Russian), Teor. Veroyatnost. $i$. Primenen 41 892-896; English translation in Theory Probab. Appl. 41 (1996), 788-791 (1977).

20. Protter, P., Stochastic Integration and Differential Equations, Springer, Berlin, 1990.

21. Rockafellar, R. T., Convex Analysis, Princeton University Press, Princeton, New Jersey, 1970.

22. SPAN (1995) Standard Portfolio Analysis of Risk, Chicago Mercantile Exchange, Chicago.

23. Wang, T. (2002) A class of dynamic risk measures, Working paper, Faculty of Commerce and Business Administration, University of British Columbia. 